\title{
Strengthening the Self-Care of Women Working in the Informal Sector: Local Fabric Weaving in Khon Kaen, Thailand (Phase I)
}

\author{
Kessarawan NILVARANGKUL ${ }^{{ }^{*}}$, Jaranya WONGPROM ${ }^{2}$, Chuanpit TUMNONG ${ }^{1}$, \\ Ausa SUPORNPUN ${ }^{1}$, Pattama SURIT ${ }^{1}$ and Niramol SRITHONGCHAI ${ }^{2}$
}

\author{
${ }^{1}$ Faculty of Nursing, Khon Kaen University, Khon Kaen, Thailand \\ ${ }^{2}$ Institute of Research and Development, Khon Kaen University, Khon Kaen, Thailand
}

Received August 31, 2005 and accepted November 25, 2005

\begin{abstract}
The first phase of this study was undertaken using Participatory Action Research (PAR) to identify the factual situations of fabric-weaving women working in the informal sector in Thailand. The aims were to enhance the mutual understanding of the women and researchers concerning the problems faced by these women and to establish an action plan to address these problems. The participant group consisted of 107 women working in the informal sector from three local villages who voluntarily took part in the study. Data were collected using quantitative and qualitative methods through structured questionnaires, focus groups, in-depth interviews, participant observations, and field notes. The results of the study revealed that the women learned their health problems were related to work and their labor potentially placed them at a risk for illness. When the women realized the gravity of their situation, they attained a sense of empowerment and took the initiative in establishing action plans to address their problems.
\end{abstract}

Keywords: Informal sector, Self-care, Weaving women, Participatory action research

\section{Introduction}

The informal sector of labor fulfills an essential role in providing jobs and reducing unemployment and underemployment. In Thailand, the number of individuals working in the informal sector is increasing due to government policies that encourage Thai people to work in their own homes or communities. For example, the government has encouraged people to establish small and medium enterprises (SMEs) by supporting their finances so that they could in such projects. It has also taken a supporting stance on the One Tambon One Product (OTOP) policy, which was designed to encourage local people in remote areas to produce their products or services using local wisdom and resources within their communities.

The estimated number of workers working in the informal sector is $20,887,400$ persons or about 69.6 percent of the employed population ${ }^{1)}$. In Thailand, the informal sector is

*To whom correspondence should be addressed. generally divided into three divisions. First, many people are outworkers employed by manufacturers but working in their homes using materials supplied by their employers. Second, some people work independently such as local fabric weavers, agricultural laborers, or traveling merchants. Finally, a number of people work in Small Scale Enterprises (SSEs).

Traditional fabric weaving is a popular source of income earning among rural women, especially in the Northeastern area because it allows them to work and tend to their families at the same time. However, weaving women often earn low incomes and lack job security. There is no work-related social welfare for them. Additionally, the women face health problems such as back, neck, arm, or hand pain due to poor postures while weaving ${ }^{2,3}$. Some women develop skin rashes from using chemical dyes and respiratory problems are common, due to the inhalation of fumes from the chemicals used to bleach silk or cotton ${ }^{2,4}$. When 40 weaving women in the Northern area of Thailand had their blood tested to identify levels of toxic metallic compounds, two samples were found to contain heavy metals ${ }^{5}$. 
Many women's health problems related to weaving are preventable if safe work practices are adopted, but the level of compliance is low. For example, the women who participated in this study regularly used chemical dyes containing high levels of metallic compounds which posed a potential health hazard. They did not wear protective gloves or masks while working with these chemicals, and as a result, the women were at risk of illness from direct and indirect contact with these toxins. The toxic dye solutions were poured on the ground without detoxification treatment after the women finished dyeing, and this resulted in a polluted environment ${ }^{2,4,6)}$.

Some initiatives for women weavers have been studied in the area of self-care, which is currently underdeveloped. This participatory action research was designed with the major aim of increasing the understanding of the processes involved in creating change and facilitating action within groups of weaving women. Rather than a top-down approach, this study used a participatory, bottom-up approach, thought which the weaving group shaped the direction of the project while the researchers facilitated this process.

\section{Materials and Methods}

This study was based on PAR principles because its main aim was to empower groups of weaving women and their communities to care for themselves and establish an ability to address their problems. The basic principles of PAR fulfill the needs of systematic collection and analyses of data to implement social or political change in those affected by the issue being studied. PAR allows researchers and participants to work along side each other in collecting data to understand the real situations of the researched population. When used effectively, it leads to heightened levels of consciousness among the participants, as well as the foundation of a collective agreement to establish plan to address their difficulties. Through involvement in the processes of data collection, decision-making, implementation, and evaluation, the researched population is given a sense of active inclusion within the research, and also a basis of empowerment to conduct the activities of the project for the purpose of addressing their own problems ${ }^{7)}$.

In this study, the research activities included recruiting participants, data collection and analyses, and reporting the results of the study.

\section{Participants}

Participants in the study were 107 women who wove cotton and/or silk in rural communities in three villages in Khon Kaen, a northeastern province in Thailand. All women were willing to participate in the study after the researchers informed and discussed the objectives, research process, and beneficial outcomes of the study with them.

\section{Research process}

This participatory action research project was divided into two phases:

Phase one (reported here) lasted one year and included:

1. Entry into the field of study

2.Data collection and preliminary data analysis

3.Development of action plans

Phase two will also require one year (the end of 2005) and focus on:

4. Implementation of the action plans

5. Evaluation

Details of phase one were as follows:

1. Entry into the field of study

The researchers initiated the study by collaborating with the village leaders, members of the Tambon Administrative Organization (TAO), local government officials, groups of weaving women, and local health personnel. After collaboration and cooperation efforts, the researchers arranged a meeting with these groups that included members of the village who were interested in this project. At the meeting, the researchers informed the village leaders and others about the objectives, processes of conducting research, and benefits of this project, allowing all interested parties to discuss various aspects of the project with the researchers. The researchers then asked for permission to conduct this project in their communities. When the researchers gained permission from the groups of weaving women, village leaders, members of TAO, and the general village members, the researchers sent an official letter to obtain permission to conduct this study from the governor of Khon Kaen.

\section{Data collection and preliminary data analysis}

\subsection{Data collection}

The researchers collected information relevant to the general statuses of the weaving women such as their age, education and income. Data regarding their history of employment and work-related illnesses over the past year were also gathered using the structured interview guidelines. Additionally, the women were physically examined by the researchers to establish information regarding their blood pressure, eyesight and muscle strength. The researchers also analyzed the components of the chemical dye.

Data relevant to the weaving women's perceptions of their risk of illness, self-care capabilities, and obstacles of selfcare were collected through focus groups, informal interviews, and participant observations. Apart from this, participant observation was used to observe the interaction among weaving women and other village members, as well as the women's posture while working. Five focus groups were conducted in each village and were audiotaped and transcribed. Informal interviews and participant observations were used through out the study. Field notes were also used 
to record information from participant observations, informal interviews, and meetings throughout the study.

\subsection{Preliminary data analysis}

During data collection to find or identify facts among the women, the researchers reflected on the emerging data and undertook a preliminary analysis with the women. This enabled the researchers and the women to discuss findings concerning the women's health problems, risks of illness, obstacles to self-care, and health needs. It also encouraged the researchers and the women to clarify each finding together. This led to a greater understanding and a valuable learning experience for both the researchers and the weaving women concerning their actual situations.

\section{Developing the action plans}

When women realized the gravity of their conditions, they set the priority of the problems by employing the Nominal Group Technique $(\mathrm{NGT})^{8}$. They then formulated five plans focusing on health promotion, illness-prevention, social welfare, and self-care education to address their problems. They also established plans to reduce the occurrence of environmental pollution within their communities.

The weaving women and researchers set meetings with people in the village to gain comments on their action plans. In addition, the women revised their action plans based on the most significant comments of these.

\section{Data analysis}

Data were analyzed using both quantitative and qualitative methods. General information such as age, education level, and income, including history and presence of illness, was analyzed using number, percentage, mean, and standard deviation statistics. The results of the physical examination, exercise, and chemical dye analyses were also analyzed using these same statistical techniques.

Weaving women's perceptions of risk of illness, self-care capabilities, obstacles to self-care, and needs of assistance were analyzed using content analysis ${ }^{9)}$. The researchers transcribed focus groups and reviewed all data collectively. The researchers extracted significant phrases, statements or sentences and categorized the data while linking relevant categories. Finally, the researchers concluded and identified themes.

Additionally, in congruency with the cyclic nature of PAR: fact-finding, planning, implementing, and evaluating, the researchers reflected on what was occurring in each phase or cycle by employing methods of description, analysis, and interpretation.

\section{Trustworthiness}

The trustworthiness of the results in this study was examined using Guba and Lincoln's (1981) ${ }^{10)}$ factors: credibility, fittingness, auditability, and confirmability. To ensure a high level of accuracy within the data, the researchers employed different methods, namely focus groups, informal interviews, participant observations, and field notes to collect data from the participants. Additionally, the researchers preliminarily analyzed data and confirmed the analyzed data with the weaving women throughout the process of the study. The researchers also returned data and their interpretation of it to the women to discuss and act upon the information during focus groups and group meetings over the sevenmonth period of data collection. The researchers also created audit trials by describing, explaining, and justifying the study's processes and the reasons behind them.

\section{Results}

To build the weaving women's capabilities of self-care, it was necessary not only to understand the actual situation of the weaving women but also for the women to become aware of their own problems, and eventually take steps to address them. In this study, the researchers reported on five issues: 1) understanding actual situations, 2) consciousnessraising, empowerment, and prioritizing the problems in order of their importance, 3 ) building leadership skills and changing the culture of depending on others, 4) developing the action plans, and 5) establishing a preliminary model to enhance weaving women's self-care.

\section{Understanding real situations}

At the beginning of the project most weaving women did not understand the nature of their real situation and had never been aware of their own problems because they had grown accustomed to their condition and perceived that this was a normal lifestyle. During the course of this project, it encouraged the weaving women, village leaders, the coresearchers comprised of the members of TAO, local health personnel, and the researchers to share their knowledge and experiences together. As a result, these groups of people now understand the real situation of the weaving women.

\section{Facing health problems related to work}

The results of the study showed that $93.5 \%$ of women had back, neck, arm, or hand pains due to poor postures while weaving. Many women suffered from these symptoms. Sixty-three percent had respiratory problems such as asthma due to cotton dust, or respiratory irritation due to the inhalation of chemicals used to bleach silk or cotton. In addition, the use of chemical dyes to color silk and cotton caused some women to develop allergic skin rashes. Six point five percent also experienced eye fatigue from weaving. Moreover, $12.1 \%$ sustained minor injuries resulting from accidents during work, such as falling from workbenches. Women also experienced stress from fear of producing fabrics of sub-standard quality, not completing orders by their 
scheduled time, and incurring debts for raw materials purchased on credit.

\section{Improper self-care}

Most weaving women lacked knowledge about how to care for themselves properly. There were no health personnel to provide them education on occupational health and safety. The women also admitted that their methods to care for themselves were not fitting to their lifestyle and culture. For example, the community practiced aerobics in the evening while the women were cooking for their families. Some women in late adulthood were also embarrassed to join an aerobics group because they had never danced in their lives. Also a few of the women's husbands did not allow them to participate in aerobics. As another example, some women used masks to prevent dust inhalation and were teased by some other villagers, which caused them to refrain from using these masks. While weaving the women remarked, "When villagers walked through my house, they teased me. For example, they said that I was being overly health conscious".

When some weaving women had neck, back, or leg pain, they used home herbal remedies. They also requested their children to step on their backs, legs, etc. as a form of pain relief. This often led to some of the women becoming bruised.

\section{At risk of illness because of working}

Many weaving women used chemical dyes containing high levels of metallic compounds such as, chromium, manganese, and lead, which posed a potential health hazard. They did not wear protective gloves or masks while working with these chemicals. As a result, the women were at risk of illness from directly and indirectly contacting these toxins. Moreover, some of the weaving women who wove cotton did not wear a proper mask to prevent inhalation of the cotton dust while weaving. Since this cotton produces substantial amount of dust, this meant they were at risk of illness in terms of respiratory ailments. Additionally, they worked with poor posture and $43 \%$ of these women had never exercised. Moreover, among the $57 \%$ of the women who did exercise, only $32.7 \%$ did so properly.

It was not only the weaving women who were at risk of illness from chemical dyes and cotton dust, but also their family members, since the women wove the fabric at their own homes. When they disposed of the packages of chemical dyes incorrectly, wind often spread the packages around the community. As the women said:

When we used the chemical dyes, we threw the packages of the chemical dyes away and our children played with the packages. Additionally, my children sometimes played with the chemically dyed water.
4. Risks of environmental pollution because of working After the women finished dyeing the materials, they poured the chemically dyed water on the ground. Before the beginning of the project most women did not know that chemical dyes were harmful to their health or the environment. They assumed that the dyes were safe because in the past their parents used these same methods when teaching them to weave fabric. After the women became involved in the project and shared their experiences and information with others, they learned that the chemical dyes were harmful for the environment. They also realized that the chemicals damaged soil and the toxicity of metal deposits in the soil might be responsible for the contamination of the underground water supply and plants. The chemical dyes also caused fish in local ponds to die.

Awareness, empowerment, and the prioritization of problems by their level of importance

When the weaving women understood the true magnitude of their situation, they became conscious of the importance of their problems and felt empowered to address them. They held a meeting to discuss their problems and they had a consensus to address the issues. As a one woman reflected:

We had never discussed our problems of weaving fabric, with others before the researchers came. After we participated in the project we came together and discussed the issues, then we learned about our problems. I then knew that we had to address these problems, especially the risk of environmental pollution, otherwise it would be harmful for our community.

Most weaving women supported this statement. Before addressing the problems, the women discussed all of their matters. They found that they were facing eight prime problems. The first of which was stress related to work. The second issue was not having any social and health welfare. The third was a lack of marketing to sell their products. The next problem was the risk of environmental pollution. The fifth problem was not engaging in exercise. No health personnel to provide education about work-related diseases and safety was the sixth problem. The seventh was improper self-care, and the final problem was depending on others rather than on themselves.

The weaving women discussed these matters with each other and concluded that they wanted to prioritize their problems, which they did using the Nominal Group Technique. The ranking of the problems was as follows, in an order of importance staring with the most important matter:

1) no social and health welfare

2) risk of environmental pollution

3) lack of exercise

4) improper self-care

5) being dependent on others rather than on themselves

6) lack of marketing to sell their products, 
7) no health personnel to provide education about work related disease and safety

8) stress related to work

After setting these priorities the weaving women, village leaders, and members of TAO identify which problems the women wanted to address. The women chose the top five ranking problems because they thought that these threatened their health and their community, and no one was addressing them. Concerning the lack of marketing to sell their products, the women concluded that the government and local politicians were helping them to amend this matter. As for stress related to work, the women perceived that they would be able to manage stress effectively by using group support and they asked for health personnel to provide education about work-related disease and safety.

\section{Building leadership capabilities}

At the start of the project, most weaving women had never discussed any noteworthy issues, including health issues, with men or others in the village. During focus group discussions, most women simply kept quiet. When they were encouraged to talk, they always said that they had the same opinion as others and often spoke bashfully. They were not able to speak publicly because they were so shy and unconfident. In addition, the weaving women always requested village leaders or the members of TAO to help them. For example, if the women wanted to address some problems or require funds to support their groups, they requested village leaders or the members of TAO to do or obtain these things for them, since they lacked knowledge and confidence to deal with their own situations. By the end of the project, most weaving women openly discussed many issues with the village men. When they shared experiences and knowledge with men, they gained knowledge on many issues from men. For example, they learned how to request funds to support their groups. Some weaving women were able to lead and encourage other weaving women to share their ideas during discussions. Most women spoke out and shared their opinions with each other during focus group discussions. Some were even able to publicly speak about their groups.

\section{Changing the culture of dependence on others}

Before the project commenced, many of the weaving women depended on others for their needs. They had never helped themselves to develop their groups. This was witnessed firsthand when some of the women asked the researchers during the project: "Do you have any money to help us. Could you please find a market to sell our products" This reflected that the women were accustomed to requesting help from others rather than taking responsibility for themselves. At the end of the project, they had changed this trend. They no longer requesting unnecessary help from others concerning their issues. The weaving women said: "From now on, we have to depend on ourselves and help each other within our group before seeking external help because we understand our own difficulties better than others".

\section{Gaining an ability to develop the action plans}

Once with the newly developed skills these women had attained in terms of independence and leadership, they were soon able to set action plans to address the problems that surrounded them in life. This sense of empowerment allowed them to hold discussions that ultimately led to the creation of five specific courses of action, as detailed below:

1. Action plan to address the lack of social and health welfare In this plan, the weaving women established a social welfare fund. The women's group collected money from its members who were willing to contribute to the fund. Then they requested the TAO to provide only $30 \%$ of the money that the fund needed. The TAO accepted and added the project to their yearly plan. This fund was founded to support its members when they were ill, disabled, or unable to work because of old age. It also supported its members by providing loans without interest when they faced financial difficulties in their families.

2. Action plan to address the risks of environmental pollution The weaving women realized that they had no way to decontaminate chemical dye solutions before disposing of it on the ground. They drew on local knowledge and advice from two scientists to devise a simple treatment system. They used dye solutions would be poured into a basket made from bamboo and enamel with some material to waterproof it. The basket was comprised of sand and rice-husk ashes in appropriate proportions. The chemical dye water would be mixed with ash to make the heavy metals coagulate. The weaving women intended to use the basket for treating chemical dye water in every household after it was tested for quality and approved by the scientists.

3. Action plan to address the problem of a lack of exercise As mentioned previously, nearly $43 \%$ of the women did not exercise due to a lack of equipment, exercise leaders for aerobic dance, and awareness of the need to exercise. The women developed a project to request equipment such as a tape player, loudspeakers, and a place within the community for the women to exercise. They also set up activities to train exercise leaders for aerobic dance and traditional stretching practices. Additionally, they established alternative activities at an appropriate time to encourage the weaving women to exercise. 
4. Action plan to address the problem of improper self-care

In this plan, the weaving women set up three activities. First, the women started a training course about self-care to prevent themselves from being exposed to work hazards. Second, they developed new methods using their local knowledge and academics to avoid coming into contact with hazardous chemicals. Finally, they provided low cost masks for weaving women to buy and use while coloring fabric and weaving cotton.

5. Action plan to address the problem of dependence on others rather than on themselves

The women set up three activities to address this problem. First, the women established a health clinic in the community to provide education about occupational illnesses and safety, as well as traditional Thai massage for their members who had body pains, and an herbal sauna. Second, the researchers trained women within the group who volunteered to provide education to other members about diseases related to work and work safety. Finally, they produced media which were suitable for them and their culture. For example, most women were undereducated, and were not fond of reading. Therefore, they provided education for their members through local songs and handbooks with more pictures than text.

\section{Developing a preliminary model of self-care enhancement for the weaving women}

Before conducting the project, the weaving women, village leaders, members of TAO, and local health personnel had never collaborated with each other in discussing their situations. After conducting the project, the results of the study established a preliminary model to enhance weaving women's self-care. This model included the process of encouraging the weaving women, village leaders, and members of TAO, local health personnel and researchers to share their experiences and knowledge freely and work with each other to identify the factual situations of the women. The process also involved encouraging democratic collaborations in making decisions. This indicated that the weaving women and community leaders identified their own problems and the action plans to address these problems.

\section{Discussion}

Regarding the reality of these women's situations, this study revealed that the weaving women in rural northeast Thailand faced many health problems such as body pain due to poor posture during work, respiratory irritation due to the inhalation of chemicals, and allergic skin rashes from using chemical dyes. These findings are in line with other studies previously conducted in Thailand ${ }^{2,3,4)}$. Furthermore, the study found that the weaving women cared for themselves improperly and were at risk for illness related to working.
Incidentally, their environment was also at risk of becoming polluted. These findings were similar to many studies ${ }^{2,4,5}$, 11). Additionally, the results of the study also indicated that the weaving women lacked social and health welfare and also earned substantially low incomes. These results are supported by other studies ${ }^{2,12,13)}$.

In the literature relating to developed and developing countries, including Thailand, no studies were found that used strategies similar to this project for developing a program to increase the self-care of weaving women.

The strategies of this project required that the women understand the reality of their situations, their problems and real causes of their problems, and then establish action plans. It was first necessary for the researchers to gain the trust of the weaving women and encourage them to develop a sense of self-worth and confidence in themselves. The researchers then employed the use of critical questions ("Why do you think, How do you feel about, etc.) to allow the women to actively share their thoughts in focus groups and develop an understanding of the real matters and bases of issues related to their self-care. In conducting the focus groups, the researchers allowed the weaving women to discuss matters freely. The open discussion in their own environment encouraged the weaving women and the community members to share their experiences, views, and knowledge with each other. This led to an ability to learn from each other and greater levels of consciousness. This idea is the heart of critical theory ${ }^{14)}$. Freire $(1974)^{15)}$ believed that critical thinking would be fostered by dialogue. The significant effect of raising individuals' consciousness is that it results in increased awareness of their own situations. When the women had their own consciousness raised, they were empowered. The problems which the weaving women accepted as their own led them to make decisions to take actions to change their own situations ${ }^{16)}$. Moreover, the researchers acted as facilitators who were willing to support and provide help, if the weaving women requested this. As a result the women gained confidence in addressing their problems.

\section{Implications}

The findings of this research revealed that there were no health personnel within the villages to provide education for the women because most occupational health personnel are employed by larger regional hospitals in metropolitan areas, rather than community public health centers. It is these public health centers, however, that cater to most persons working in the informal sector. These findings stand as a strong testament to the need for the Department of Workrelated Disease and Environment, Ministry of Public Health to establish positions of occupational health within public health centers. The Department of Labor Protection and 
Welfare, Ministry of Labor is also encouraged to contribute funds for the establishment of a social welfare program for those working in the informal sector, because those currently employed in jobs such as this receive very few benefits from their local governments. Information concerning policies related to environmental protection should be widely propagated and these policies should be strictly enforced to encourage adherence by weaving women.

\section{Conclusion}

This study constituted the early stage of building the weaving women's capacity by encouraging them to understand the seriousness of their own situations. This led to the women to be aware of their matters and feel empowered. They then made decisions together to developing action plans to address the issues. The women also want to be trained as agents for change to strengthen their groups in self-care. However, to ensure an accurate perspective of the benefits and nature of the action plans within this study, a concise implementation and evaluation of them will be necessary in the second phase of this research effort.

\section{Acknowledgments}

My sincere thanks go to all who participated in this research. My heartfelt thanks also go to the Thailand Research Fund for providing financial support for the fulfillment of this project.

\section{References}

1) National Statistics, Prime Ministry Office (1999) Surveying home-based work. P.A.Liveing Company, Bangkok.

2) Nilvarangkul K, Wongprom J, Pinchareon S, Thanattherakul C (2004) Assessing the health impact of the One Tambon One Product policy on local fabric weaving groups in North-Eastern Thailand. Journal of Nurses' Association of Thailand north-eastern Division, 22, 57-65.
3) Jamratphun W, Wongprom J, Sunthornchai T, Srithongchai $\mathrm{N}$ (1999) A study of paying women working in the informal sector. Research and Development Institute Khon Kaen University, Khon Kaen.

4) Supapornhemin S (1999) Weaving fabric for environment: experience and lesson learn from abroad. n.p.

5) The Study and Development of Home-Based Work in the Northern Region (1997) Surveying Basic Information of the Groups of Home-Based Workers. 38-39, Homenet Thailand, Chiangmai.

6) Mahachai R, Phuwongpha N (2003) The treatment of effluent form dye industries by using rice-husk ashes. Department of Chemical the Faculty of Science Khon Kaen University, Khon Kaen.

7) Whyte WF (1991) Participatory action research. Sage, Newbury Park, California.

8) Brchan MS, Hegge MJ, Stenvig TE (1991) A tigger by the tail: tackling barriers to differentiated practice. The Journal of Continuing Education in Nursing 22, 109-12.

9) Berelson B (1971) Content analysis in communication research. Hafner Publishing Company, New York.

10) Guba EG, Lincoln YS (1981) Effective evaluation. JosseyRass Publication, San Fransico.

11) Natapintu K, Keskowit K, Kanjanopass P, Natapintu S, Intarassamee J, Ritmaak P, Pongwijit M (2001) A study and development a model of learning and solving occupational health problems at home-based work in the Northeast Thailand. The Faculty of Public Health, Khon Kaen University, Khon Kaen.

12) Yim Yam S, Meesid C, Udomwong N, Leechanawanitchawun R (2000) The synthesis of knowledge on informal workers. Chiangmai University, Chiangmai .

13) Wongprom J, Sunthornchai T, Yenbumrong T, Srithongchai N (2004) How-based workers: data and situation in four regions. Research and Developing Institute Khon Kaen University, Khon Kaen.

14) Harbermas J (1971) Knowledge and human interest. Beacon Press, Boston.

15) Friere P (1974) Pedagogy of the oppressed. The Seabury Press, New York.

16) Wallerstein N (1992) Powerless, empower, and health: implications for health promotion. Am J Health Promotion 6, 197-205. 